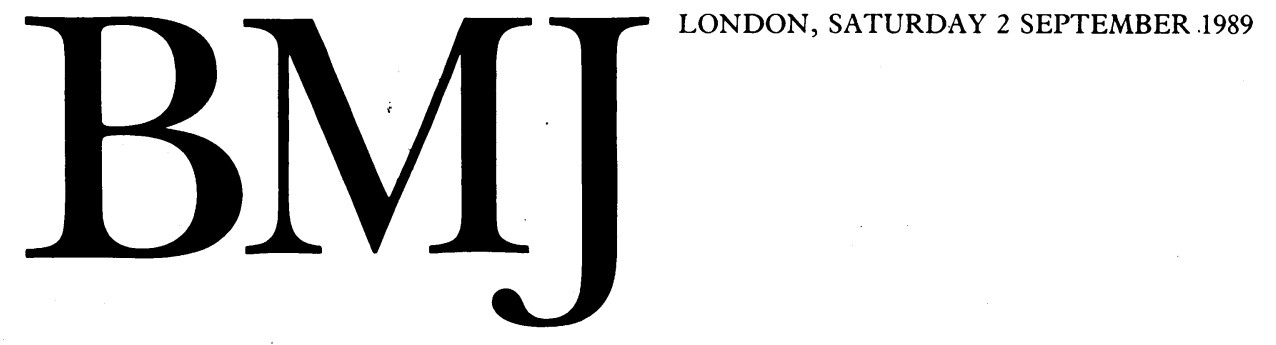

\title{
Desktop laboratory technology in general practice
}

\author{
Quality assurance schemes are important
}

Technology is invading general practice at a rapid rate. Peak flow meters, electrocardiographs, electronic thermometers, tonometers, audiometers, haemoglobinometers, sonicades, dipsticks for biochemical or microbiological testing, biochemical desktop analysers, pregnancy tests, endoscopes, and even computer assisted clinical decision making are changing the scene in the hitherto low tech family doctor's consulting rooms. ${ }^{1}$ Unfortunately, market forces can be as influential as scientific reasoning when some choices are made so the risks, benefits, and opportunity costs of new technology in primary health care will need critical appraisal over the next decade if the best interests of patients are to be served.

Assays of blood cholesterol concentration illustrate some of the issues because population screening for cholesterol has special appeal to those who market computers, desktop analysers, and lipid lowering drugs. A programme targeted at adults aged over 20 and taking a cut off point for plasma cholesterol concentration of $6.2 \mathrm{mmol} / 1$ would result in about half the British population needing an annual review ${ }^{2-4}$; if $2 \%$ of those screened ever resorted to lipid lowering drugs the pharmaceutical costs could be staggering. It is therefore salutary to remember that the scientific case for population screening is still challenged by authoritative groups, ${ }^{4}$ and general practitioners can be justifiably cautious about acquiring their own lipid desktop analysers and embarking on population screening programmes until the adverse effects of lipid screening are better known.

Desktop biochemical analysis raises another important issue for enthusiastic practitioners - that of quality control. Responsibility for the validity of biochemical results has hitherto lain with laboratory based biochemists, but once tests are performed outside laboratories the responsibility passes to the practitioners. A recent report drew attention to the fact that between instrument dispersion of results from plasma cholesterol assays is 1.3 times greater with general practitioners' desktop analysers than with hospital laboratory instruments. ${ }^{5}$ Nearly $10 \%$ of the results in general practice deviated by $1.0 \mathrm{mmol} / \mathrm{l}$ from the test standards. Repeating tests on individual patients in a misguided attempt to compensate for such variability is uneconomic, incompatible with patient centred care, and prone to a drift towards mean values, which themselves may be erroneous for methodological reasons.

Research into monitoring of blood glucose concentrations at home, in the clinic, or in the ward using reagent strips and reflectance meters has also shown that unacceptable errors are three times more likely than with laboratory based assays despite the excellent optical precision of the meters. ${ }^{6-9}$ These are usually due to improper calibration, improper application of the sample to the reagent strip, build up of cotton fibres in the optical compartment after wiping, incorrect location of the strip in the meter, contamination of the optical compartment with blood, defective or out of date reagent strips, and electrical faults. ${ }^{10}$ All are preventable by paying attention to detail and quality control checking.

Regular calibration and rigorous quality control checks on all diagnostic equipment are familiar to full time hospital laboratory staff, ${ }^{11}$ but in general practice fewer assays are done so both doctors and nurses are likely to use the equipment intermittently and to have little or no training in laboratory quality assurance. One practice that employs a part time laboratory technician found its family practitioner committee reluctant to reimburse $70 \%$ of the costs under red book regulations. Yet reliance on a test that is not of proved reliability can prejudice a life assurance application, a preemployment medical examination, or the accuracy of a diagnosis, with both medicolegal and clinical consequences. These considerations will be even more important in the new competitive NHS of the 1990s, in which budgets, internal markets, and cost cutting will be encouraged and many practitioners will be tempted by the image or economy of doing more tests in their practices.

What then should general practitioners do when tempted to install their own desktop diagnostic equipment? Firstly, be vigorous in justifying the investment and its use. Do not be bullied by offers from pharmaceutical computer salesmen, pressure from the regional adviser to update the practice, or assertions from the nurse that "everyone is doing it." Next, discuss the idea with the head of the local hospital laboratory to ensure its support in establishing training for the practice staff and back up for internal and external quality control of all assays with further investigation when indicated. Finally, count the cost of the machine, staff time, maintenance, reagents, and insurance cover.

All NHS hospital laboratories in the United Kingdom participate in an external quality control scheme, which is overseen by a professional watchdog group and a Department of Health advisory committee. Participation is voluntary and confidential, but an annual report of the grouped results makes fascinating reading and provides peer review of the 
best kind. " Recently the Wolfson Research Laboratories have invited all general practitioners with desktop instruments for measuring plasma cholesterol concentration to participate in a national quality assessment programme ${ }^{12}$; local biochemistry or haematology laboratories will be in a good position to provide advice about other schemes and back up services.

Desktop microanalysers have come of age, but the far reaching consequences of their uncontrolled use have not been fully grasped by the early adopters of this new technology. If a new technology is really essential in modern primary health care it must be introduced in a patient centred way that researches the risks, benefits, and opportunity costs so that what the government calls "customers" can at least know that their doctors are striving for an informed professional view of what is in their best interests and applying it responsibly. Internal and external quality control of biomedical assays and the risks of cross infection from other diagnostic equipment are important issues for general practitioners and primary care nursing staff today.

Professor of General Practice,

N C H STOTT

University of Wales College of Medicine,

Cardiff CF3 7PN

1 Neville RG. Introduction of new diagnostic aids in general practice. Update 1988;36:2311-5.

2 National Cholesterol Education Program. Report of the expert panel on detection, evaluation and treatment of high blood cholesterol in adults. Arch Intern Med 1988;148:36-9.

3 Shaper AG, Pocock SJ. Risk factors for ischaemic heart disease in British men. British Health fournal 1987;57:11-6.

4 Smith WCS, Kenicer MB, Maryon-Davies A, Evans AE, Yarnell J. Blood cholesterol: is population screening warranted in the UK? Lancet 1989;i:372-3.

Broughton PMG, Bullock DG, Cramb R. Quality in plasma cholesterol measurements in primary care. Br Med F 1989;298:297-8.

6 Petranje G, Petranje M, Scobie IN, et al. Quality control of home monitoring of blood glucose concentrations. BrMed f 1984;288:757.

7 Holloran SP. Experience with Reflolux 11/BM 1-44 glycerine stick blood glucose meter system. Ann Clin Biochem 1987;24:168-9.

8 Rady AA, Jones KW, Holloran SP, Marks V. Bedside blood glucose measurement: quality control and assessment. Ann Clin Biochem 1987;24(suppl 1):170-1.

and assessment. Ann Clin Biochem 1987;24(suppl 1):170-1.
9 Flynn BJ. The quality assessment of blood glucose assays outside the laboratory-one year's experience. Ann Clin Biochem 1987;24(suppl 1):172-3.

10 Jones KW, Rady A, Holloran SP, Marks V. A system to improve the standard of bedside blood glucose measurements-its introduction and management. Ann Clin Biochem 1987;24(suppl 1):174-5.

11 Advisory Committee on Assessment of Laboratory Standards. United Kingdom external quality assessment schemes annual report 1987. London: DHSS, 1987.

12 Bullock DG, Broughton PMG. Cholesterol assays in primary care. Horizons 1988;2:700.

\section{Good clinical practice: a way to better drugs}

\section{Rules for clinical drug research that offer a prospect of better trials}

When drug regulatory authorities use the term "good clinical practice" clinicians may fear more bureaucratic constraints. No such assault is intended. Good clinical practice signifies a set of rules for performing standards and helping to encourage cross national acceptance of data from trials for regulation. The need for standards for performing clinical trials has grown as clinical evaluation of drugs has become more complicated. Poorly performed trials are still carried out and presented to regulatory agencies in support of new drug applications. The pharmaceutical industry, which sponsors most new drug trials, has therefore issued various good practice guidelines, ${ }^{1}$ and some national drug agencies-for example, in France, Japan, and the United States - have done the same. Over a decade ago the Food and Drug Administration worked out a large complex of regulatory requirements for clinical testing of investigational drugs, ${ }^{2}$ and the European Community is about to publish general recommendations on the conduct of clinical trials. ${ }^{3}$ Both the European Community and the Nordic Council of Medicines are presently drafting guidelines on good clinical practice.

Progress in the methods of trials and the general acceptance of ethical responsibility ${ }^{4}$ have stimulated this development, but just as important are political and commercial factors. Thus public and political concerns over widely published examples of research fraud, accidents during trials, and questionable ethics may be overdramatised but are not entirely unwarranted. Furthermore, to exploit the huge markets for drugs in the European Community, United States, and Japan pharmaceutical companies require that data from trials in one country are accepted in others. The member states must therefore also harmonise their own regulations. The good clinical practice guidelines being drafted by the European Community's committee on proprietary medicinal products and due to be published in early 1990 are a part of this initiative.

What does a good clinical practice guide contain? The likely construction of the European Community document may give some idea. It will include $(a)$ a detailed glossary explaining all the terms used in the document to avoid misunderstandings; (b) instructions protecting subjects taking part in the trial, in line with the Declaration of Helsinki, covering both ethics committees and informed consent; $(c)$ requirements for "standard operating procedures" explaining in detail the responsibilities of the sponsor (usually a pharmaceutical company), the investigator (the clinically responsible doctor), and the monitor (the sponsor's trial supervisor) before, during, and after the trial; $(d)$ instructions for drawing up protocols, reporting to the authorities - for example, adverse events - data handling and archiving, and statistics, etc; and (e) requirements for auditing, data verification, and quality control. The guidelines serve as a checklist to ensure that all those taking part in clinical drug research know exactly what is required of them. Those concerned should carefully adhere to the standards and procedures if they want the data produced to be used in applications for marketing authorisations. In this way dependable and verifiable data of the highest scientific and ethical quality may be generated. Although good clinical practice guidelines are primarily directed towards trials initiated by the pharmaceutical industry, their impact on the conduct of trials in general should not be underestimated.

The document now being drafted will not have the force of law when published, but it will carry considerable implications for those conducting clinical trials in Europe. Perhaps most important is the fact that investigators will be controlled and trials monitored to an extent that only few have experienced. In fact inspectors for the Food and Drug Administration have long visited investigators and sponsors in Europe as well as in the United States, and the French regulatory authority has already started doing the same. Such operations may be contrary to the traditions of European research, but verification of data should not be confused with interference with the free practice of medicine. Nevertheless, if a study is not performed in accordance with good clinical practice or the data cannot be verified then the study will not be accepted as part of a regulatory submission. As the demand for good clinical practice is essentially a demand for quality it should increase investigators' carefulness and prevent bungling and fraud. In turn, this may affect positively the practice of medicine in general and of drug treatment in particular.

Will the good clinical practice requirements help us to obtain better treatments? They probably will, but on two conditions: the trial regulations should not interfere unduly 